\title{
Patient-Tailored In Silico 3D Simulations and Models From Electroanatomical Maps of the Left Atrium
}

\author{
Gonzalo R Ríos-Muñoz ${ }^{1,2}$, Sara Rocher ${ }^{3}$, Antonio Artés-Rodríguez ${ }^{1,2}$, Ángel Arenal ${ }^{2}$, Javier Saiz ${ }^{3}$, \\ Carlos Sánchez ${ }^{4}$ \\ ${ }^{1}$ Universidad Carlos III de Madrid, Leganés, Spain ${ }^{2}$ IiSGM, Madrid, Spain \\ ${ }^{3} \mathrm{Ci}^{2} \mathrm{~B}$, Universitat Politècnica de València ${ }^{4}$ CUD, BSICoS, CIBER-BBN, Zaragoza, Spain
}

\begin{abstract}
The mechanisms underlying atrial fibrillation $(A F)$ are still under debate, making treatments for this arrhythmia remain suboptimal, with most treatments applied in a standard fashion with no patient personalization. Recent technological advances in electroanatomical mapping (EAM) using multi-electrode catheter allow the physicians to better characterize the substrate, thanks to a better spatial resolution and higher density of acquisition points. Taking advantage of this technology, we describe a workflow to build personalized electrophysiological atrial models for $A F$ patients. We seek to better predict the outcome of a treatment and study the AF problem in a more specific scenario. We generated physiological $3 D$ models from the EAM data using hexahedral meshing of element size $300 \mu \mathrm{m}$, and added fiber orientation based on a generic model. We used the local activation time (LAT) maps performed in sinus rhythm (SR) to estimate the conduction velocity $(C V)$ of the regions in the atrium with a new method that combines the LATs of neighboring tissue as the average $C V$ of triplets of points. We also characterized the cellular model by Maleckar et al. in terms of longitudinal conductivity and $C V$ to personalize the atrial models. We were able to simulate $S R$ and AF scenarios on the personalized models, and we generated a database of atrial models for future analysis.
\end{abstract}

\section{Introduction}

Atrial fibrillation (AF) is the most common cardiac arrhythmia in the clinical practice. However the mechanisms initiating and maintaining AF are still under debate, preventing electrophysiologists from finding an optimal treatment to terminate this arrhythmia. Most treatments are based on routine protocols applied without taking into account differences in the substrate (scars, fibrotic areas), or signal related characteristics (voltage, dominant frequency, reentrant mechanisms, rotors) of the patients.
Under these circumstances in silico patient specific models have proven to be useful, combining both electrical and anatomical features with mathematical models. The simulation approach accounts for tissue heterogeneity, as well as different cellular models and anatomies, which allow in silico simulations to replicate arrhythmia scenarios. This offers physicians the opportunity to study the effects of drugs or ablation lesions, predicting the therapy outcome in a controlled environment.

For this reason the data directly acquired from the patients plays an important role in the personalization of these models. Many data sources are available, i.e., surface electrocardiograms, intracardiac electrograms, MRI scans, and also electroanatomical maps. The later offers both geometrical reconstruction of the heart chambers and substrate characterization by using multi-electrode catheters to guide physicians during ablation procedures. However, all this information usually remains unused after the clinical procedure is done. Our objective is to reuse the EAM information to spatially characterize the simulated tissue. With an accurate 3D reconstruction of the heart chamber and its electrical behavior, we can adjust cellular level models and include fiber orientation, providing heterogeneous anatomical models that mimic the electrophysiological characteristics of the patient.

\section{Methods}

\subsection{Electroanatomical Data}

We collected the data for the study from 28 persistent $\mathrm{AF}$ patients who underwent first ablation protocol at the Hospital General Universitario Gregorio Marañón. All patients gave informed consent and the study was approved by the Institutional Review Board (IRB) of the center. The maps were generated using a multi-electrode catheter PentaRay and CARTO EAM system (Biosense Webster). We exported and post-processed the maps to obtain 3D geometries of $600 \mu m$ thickness and regular hexa- 

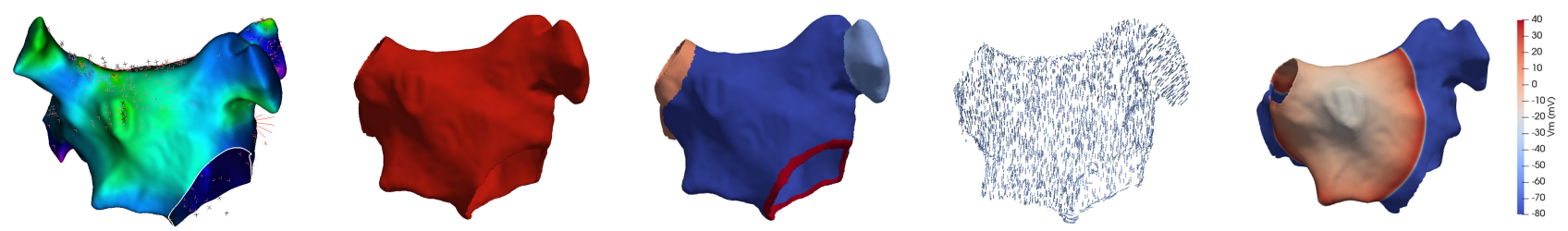

Figure 1. Model personalization workflow. From left to right: electroanatomical map, hexahedral geometry, region segmentation, fiber orientation, and simulation with personalized $\mathrm{CV}$.

hedral elements of $300 \mu \mathrm{m}$. The anatomy was manually segmented by electrophysiologists, pulmonary veins and mitral ring removed, and atrial appendage was preserved (see workflow in Figure 1). The electroanatomical voltage maps of the different left atria were generated in both $\mathrm{AF}$ and SR, with additional LAT map performed on the latter.

\subsection{Estimation}

From the LAT propagation maps, we can select two points $A$ and $B$ with known LATs, namely $L A T_{A}$ and $L A T_{B}$, and compute the $\mathrm{CV}$ as

$$
C V=\frac{\operatorname{dist}(A, B)}{\left|L A T_{B}-L A T_{A}\right|}
$$

where $\operatorname{dist}(A, B)$ is the Euclidian distance between two points. However, this approach does not take into account the direction of the wavefront and the results could be misleading, obtaining $\mathrm{CV}$ values outside the physiological range. As a requirement, at least three non-collinear points are needed to calculate the $\mathrm{CV}$, i.e., a triplet of points (see Figure 2). Methods based on this triangulation approach have been already proposed in the literature [1-4], however most of them place the triplet with prior knowledge of the direction of activation, or the layout of the electrodes in the catheter.

Our method estimates both the $\mathrm{CV}$ and the propagation direction without prior direction knowledge. We calculate the $\mathrm{CV}$ at a point $P$ given the LATs of the triplets comprising the neighboring points that belong to the circumference of radius $r$ and center $P$ (see Figures 3 and 4). We use a circumference to avoid $\mathrm{CV}$ calculation of close points that may produce abnormal $\mathrm{CV}$ values beyond the physiological range. On the other hand, having too distant points may not accurately represent the local tissue $\mathrm{CV}$ at point $P$, so we set $r=1 \mathrm{~cm}$ given the size of the atrium. We select beforehand the number of points from the circumference that we use, namely $N$. The circumference is tangent to the atrial wall at point $P$, with its $N$ points equally distributed. Since atrial tissue is highly irregular, this prevents an optimal matching with the proposed circumference, the circumference points $n_{i}$, where $i \in[1, N]$, need to be corrected so that they belong to the atrial geometry.

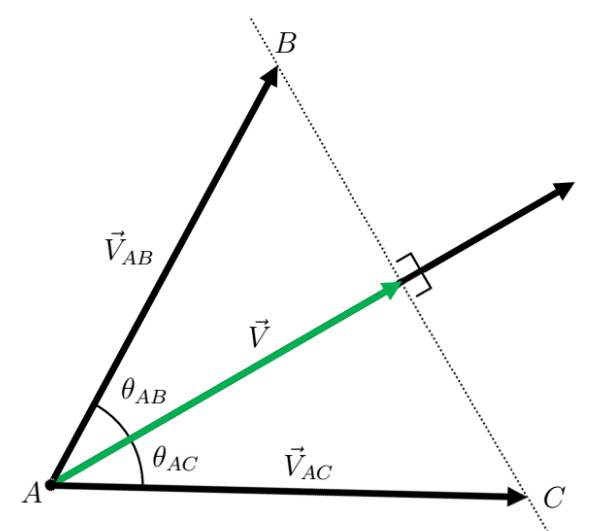

Figure 2. Calculation of the CV vector $\vec{V}$ using the scalar projection of the velocity vectors $\vec{V}_{A B}$ and $\vec{V}_{A C}$. Points $A$, $B$, and $C$ constitute a triplet, with known spatial coordinates and LATs.

With the circumference points we build a total of $T=$ $N$ triplets, forcing the central point $P$ to be in every triplet. Figures 3 and 4 provide an illustrative example. Triplet points should satisfy a minimum distance constraint $\operatorname{dist}\left(n_{i}, n_{i+1}\right) \geq d_{m i n}$, where $d_{m i n}=1 \mathrm{~mm}$ is given by the spatial resolution of the EAM system. Additionally we set the maximum allowed angle for the triplet vertices, namely $\theta_{\text {max }}$.

Once the triplets have been defined, we estimate a CV vector $V_{i}$ for each triplet, where $i \in[1, T]$ refers to the $i$-th triplet index. For a given triplet of points $A, B$, and $C$ defining the triangle $A \hat{B} C$ (Figure 2), we estimate the velocity vector $\vec{V}_{i}=\left[V_{x}, V_{y}, V_{z}\right]$ as the scalar projection of the velocity vectors $\vec{V}_{A B}=\left[V_{A B x}, V_{A B y}, V_{A B z}\right]$, and $\vec{V}_{A C}=\left[V_{A C x}, V_{A C y}, V_{A C z}\right]$ [5]. Solving the equation

$$
\|\vec{V}\|=\operatorname{proj}_{\vec{V}} \vec{V}_{A B}=\operatorname{proj}_{\vec{V}} \vec{V}_{A C},
$$

and using trigonometrical identities, we can derive the following system of equations to calculate the value of $\vec{V}$,

$$
\left\{\begin{array}{rr}
V_{A B x} \cdot V_{x}+V_{A B y} \cdot V_{y}+V_{A B z} \cdot V_{z}= & \Upsilon_{A B}, \\
V_{A C x} \cdot V_{x}+V_{A C y} \cdot V_{y}+V_{A C z} \cdot V_{z}= & \curlyvee_{A C}, \\
\alpha \cdot V_{x}+\beta \cdot V_{y}+\gamma \cdot V_{z}= & 0,
\end{array}\right.
$$




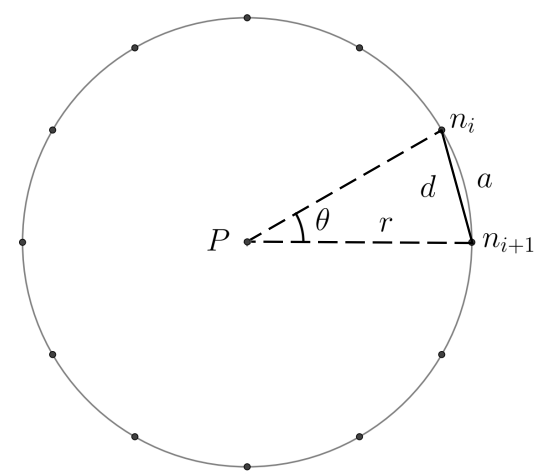

Figure 3. Triplet configuration to calculate the $\mathrm{CV}$ at reference point $P$, where $r$ the circumference radius, $n_{i}$ and $n_{i+1}$ consecutive points in the circumference, $a$ the arclength between points $n_{i}$ and $n_{i+1}, d$ the length of the chord connecting $n_{i}$ and $n_{i+1}$, and $\theta$ the angle defined by the triangle $\left[n_{i}, P, n_{i+1}\right]$.

where

$$
\begin{aligned}
\Upsilon_{A B} & =\|\vec{V}\| \cdot\left\|\vec{V}_{A B}\right\| \cdot \cos \left(\theta_{A B}\right), \\
\curlyvee_{A C} & =\|\vec{V}\| \cdot\left\|\vec{V}_{A C}\right\| \cdot \cos \left(\theta_{A C}\right), \\
\alpha & =\left[V_{A B y} V_{A C z}-V_{A B z} V_{A C y}\right], \\
\beta & =\left[V_{A B z} V_{A C x}-V_{A B x} V_{A C z}\right], \\
\gamma & =\left[V_{A B x} V_{A C y}-V_{A B y} V_{A C x}\right] .
\end{aligned}
$$

Then, we average the contribution of all the CV vectors $\vec{V}_{i}$, obtaining an estimation of the $\mathrm{CV}$ as

$$
\vec{V}=\frac{1}{T} \sum_{i=1}^{T} \vec{V}_{i}
$$

The direction of the wavefront is also estimated since the 3 components of the CV vector $\vec{V}$ are obtained. As a remark, point $A$ is assigned to the point of the triplet with the earliest LAT. In the event of two simultaneous LATs, $A$ is assigned to the latest LAT and the resulting CV vector is multiplied by -1 .

\subsection{Personalized Model Parameters}

The CV depends on fiber orientation, model geometry, tissue conductivity, and wavefront curvature among other factors. For the simulations, we use the cellular model by Maleckar et al. [6]. To characterize the model and the chosen geometry, we generated a regular hexahedral grid of size $L \times W \times H=6 \times 3 \times 0.06 \mathrm{~cm}$ with element size $300 \mu \mathrm{m}$. We applied a periodic $500 \mathrm{~ms}$ stimulation train and measured the $\mathrm{CV}$ between 2 points at both edges of the grid in the propagation direction. We characterized the $\mathrm{CV}$-conductivity function by varying the value of $\sigma_{L}=\left[10^{-4}, 5 \cdot 10^{-4}, 10^{-3}, 2 \cdot 10^{-3}, 3 \cdot 10^{-3}, 4 \cdot 10^{-3}, 5\right.$.

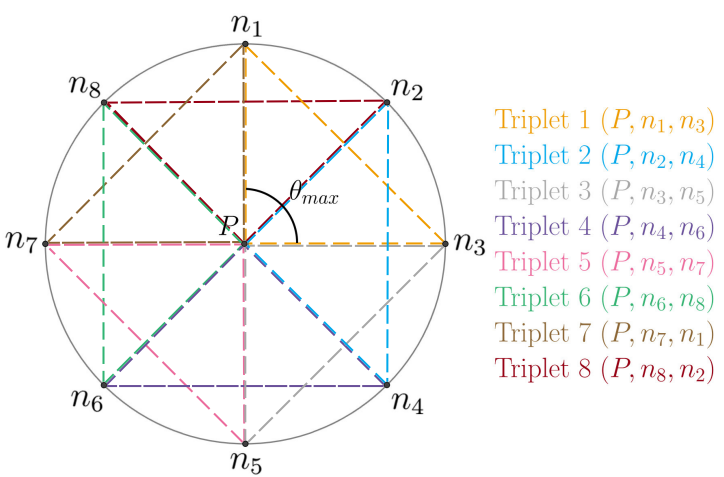

Figure 4. Triplet combinations for $N=8$ circumference points, and maximum angle at the $P$ vertex $\theta_{\max }=90^{\circ}$.

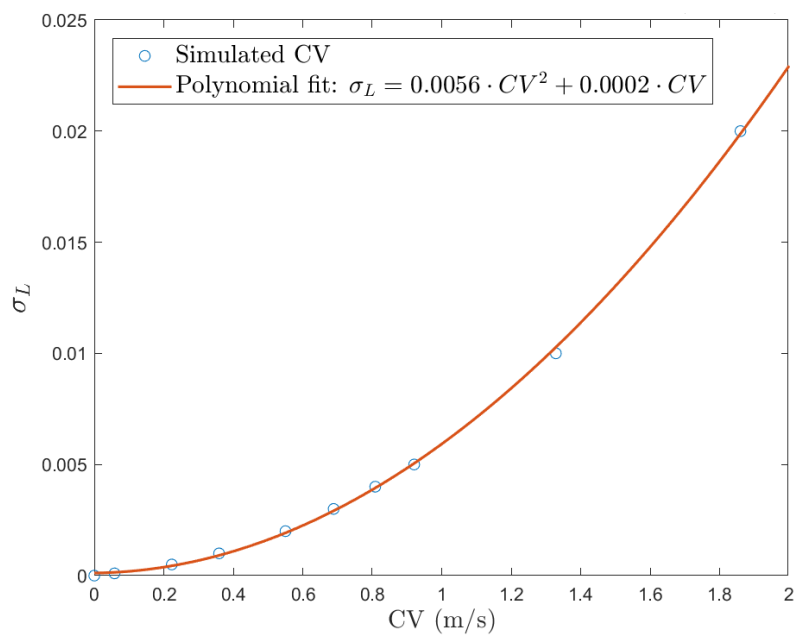

Figure 5. CV characterization for hexahedral elements of size $300 \mu \mathrm{m}$ using Maleckar et al. cellular model [6]. Given longitudinal conductivity values, $\sigma_{L}$, and CV computed from simulations we fit a second order polynomial.

$\left.10^{-3}, 10^{-2}, 2 \cdot 10^{-2}\right] \frac{S}{\mathrm{~cm} \cdot \mathrm{pF}}$ (higher values resulted in no propagation). We fitted the data to a second order polynomial as illustrated in Figure 5.

For the whole left atrium, we spatially calculate the $\mathrm{CV}$ for different atrial regions at $1 \mathrm{~cm}$ equally distributed points, using interpolation to fill the rest of the anatomy. We include pre-existing fiber orientation using a ruledbased method to complete the atrial model personalization [7].

\subsection{Simulations}

In silico simulations were run using ELVIRA, a finite element method-based software [8]. The integration time step was set to $0.01 \mathrm{~ms}$ and the acquired action potential signals in the grid nodes and complete atrial models were sampled at $1 \mathrm{KHz}$. 


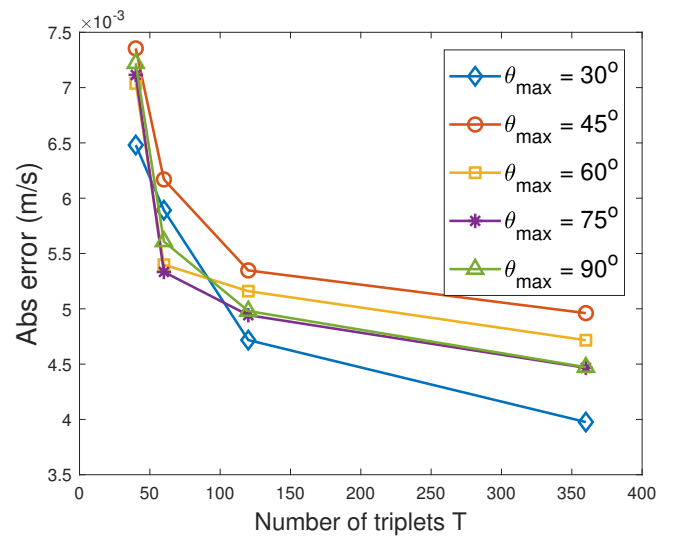

Figure 6. Absolute error results for different $\theta_{\max }$ and $T$ values. Ground truth was calculated in the rectangular grid simulations and averaged for the different values of $\sigma_{L}$ used to fit the CV-conductivity function in Figure 5.

\section{Results}

We analyzed the new CV estimation method to characterize its properties in terms of the number of triplets $N$ and the maximum allowed angle $\theta_{\max }$ in the simulated grid under controlled conditions and in experimental data directly measured from the EA maps. Performance of the method is shown in Figure 6. From the results we can conclude that with this method the obtained $\mathrm{CV}$ values are very similar to the ones in the grid and experimental data. We see how by increasing the number of triplets the estimation of $\mathrm{CV}$ improves, and that the best performance is achieved for $\theta_{\max }=30^{\circ}$.

Additionally, we managed to integrate the CV estimation into the geometrical model. We achieved to run simulations replicating SR activation and also AF after overstimulation at different points of the atrium, e.g. near the pulmonary veins and atrial appendage.

\section{Discussion and Conclusions}

The CV estimation method presents promising results to estimate both the magnitude of the $\mathrm{CV}$ and the direction of the wavefront in the cardiac tissue. We built a database of personalized atrial models in a straightforward way. This database could be used to test clinical treatments, new signal processing techniques, and validation methods as in $[9,10]$. Further validation is to be done, including additional tissue properties, e.g. voltage information, or fibrosis from MRI scans. The generated atrial model database is expected to be increased with forthcoming data.

\section{Acknowledgements}

This work has been partly supported by MINECO/FEDER (ADVENTURE, id. TEC2015-69868-C2-1-R), Co- munidad de Madrid (CASI-CAM-CM, id. S2013/ICE2845), Centro de Investigación Biomédica en Red (CIBER), proyecto DPI2016-75458, and the Programa Prometeo, Generalitat Valenciana, Award Number: 2016/088.

\section{References}

[1] Corrado C, Williams S, Karim R, Plank G, O'Neill M, Niederer S. A work flow to build and validate patient specific left atrium electrophysiology models from catheter measurements. Medical Image Analysis 2018;47:153-163.

[2] Krueger MW, Dorn A, Keller DUJ, Holmqvist F, Carlson J, Platonov PG, Rhode KS, Razavi R, Seemann G, Dössel $\mathrm{O}$. In-silico modeling of atrial repolarization in normal and atrial fibrillation remodeled state. Medical and Biological Engineering and Computing 2013;51(10):1105-1119.

[3] Cantwell CD, Roney CH, Ng FS, Siggers JH, Sherwin SJ, Peters NS. Techniques for automated local activation time annotation and conduction velocity estimation in cardiac mapping. Computers in Biology and Medicine 2015; 65:229-42.

[4] Verma B, Oesterlein T, Loewe A, Luik A, Schmitt C, Dössel $\mathrm{O}$. Regional conduction velocity calculation from clinical multichannel electrograms in human atria. Computers in Biology and Medicine 2018;92:188-196.

[5] Zheng Y, Xia Y, Carlson J, Kongstad O, Yuan S. Atrial average conduction velocity in patients with and without paroxysmal atrial fibrillation. Clinical Physiology and Functional Imaging 2017;37(6):596-601.

[6] Maleckar MM, Greenstein JL, Giles WR, Trayanova NA. $\mathrm{K}+$ current changes account for the rate dependence of the action potential in the human atrial myocyte. AJP Heart and Circulatory Physiology 2009;297(4):H1398-H1410.

[7] Ferrer A, Sebastián R, Sánchez-Quintana D, Rodríguez JF, Godoy EJ, Martínez L, Saiz J. Detailed anatomical and electrophysiological models of human atria and torso for the simulation of atrial activation. PLOS ONE 2015; 10(11):e0141573.

[8] Heidenreich EA, Ferrero JM, Doblaré M, Rodríguez JF. Adaptive macro finite elements for the numerical solution of monodomain equations in cardiac electrophysiology. Annals of Biomedical Engineering 2010;38(7):2331-2345.

[9] Ríos-Muñoz GR, Arenal Á, Artés-Rodríguez A. Real-time rotational activity detection in atrial fibrillation. Frontiers in Physiology 2018;9:208.

[10] Sánchez C, Bueno-Orovio A, Pueyo E, Rodríguez B. Atrial fibrillation dynamics and ionic block effects in six heterogeneous human $3 \mathrm{~d}$ virtual atria with distinct repolarization dynamics. Frontiers in Bioengineering and Biotechnology 2017;5:29.

Address for correspondence:

Gonzalo R. Ríos-Muñoz

Universidad Carlos III de Madrid

Department of Signal Theory and Communications Lab. 4.3.B01 Avda. de la Universidad, 3028911 Leganés (Madrid), SPAIN griosm@tsc.uc3m.es 